\title{
Severe Fever with Thrombocytopenia Syndrome Virus Replicates in the Brain Tissue and Damages Neuron in Newborn Mice
}

\section{Rui Chen}

Wuhan University

Qiang Li

Wuchang University of Technology

Hongmei Chen

Wuchang University of Technology

Hongguang Yang

Wuhan University

Xuemin Wei

Shandong University)

Mengting Chen

Shandong University)

Hongling Wen ( $\nabla$ wenhongling@sdu.edu.cn )

Shandong University)

\section{Research Article}

Keywords: SFTSV replicate, brain, neuron

Posted Date: January 19th, 2022

DOI: https://doi.org/10.21203/rs.3.rs-1143683/v1

License: (9) (i) This work is licensed under a Creative Commons Attribution 4.0 International License. Read Full License 


\section{Abstract}

Severe fever with thrombocytopenia syndrome (SFTS) virus (SFTSV) is a emerging tick-borne phlebovirus with a high fatality rate of $12-30 \%$, which has an expanding endemic and causes thousands of infections every year. Central nervous system (CNS) manifestations are an important risk factor for the death outcome of SFTS. In-depth understanding of the process of SFTSV invading the brain is critical for developing effective anti-SFTS encephalitis therapeutics. Here, we reported the change of viral load of brain at different time points after intraperitoneal infection of SFTSV in newborn C57/BL6 mice. The virus invaded the brain at 3 hours post-infection (hpi). Notably, the viral load increased exponentially after 24 hpi. In addition, we reported that in addition to macrophages, SFTSV infected neurons and replicated in brain. These findings provide insights into the CNS manifestations of severe SFTS, which may aid in drug development and encephalitis therapeutics.

\section{Introduction}

Ticks are the second largest transmission vector of pathogens after mosquitoes[1]. Infectious diseases transmitted by ticks are a growing threat to global public health[2]. Severe fever with thrombocytopenia syndrome (SFTS) is an emerging tick-borne infectious disease caused by SFTS virus (SFTSV) with a mortality rate of $6-30 \%[3]$. SFTS was first recorded in China in 2009, followed by reports in South Korea, Japan, and Vietnam[3-6]. At present, SFTS cases are gradually increasing in these countries. As of 2018, a total of 11995 cases had been reported in China alone[7]. Haemaphysalis longhorned tick is the main transmission vector of SFTSV, which is widely distributed in Asia, Oceania and North America[8, 9]. Epidemiological investigations showed that close contact with blood/bloody secretions of SFTS patients can also cause human-to-human transmission[10, 11]. In addition, SFTSV RNA was detected in sputum and semen, suggesting that droplets and sexual transmission may be potential transmission routes[12, 13]. Our previous study found that SFTSV could be transmitted vertically through the placental barrier in C57/BL6 mice[14]. All these findings suggest that human-to-human transmission may have multiple transmission routes.

SFTS patients show a variety of clinical manifestations, including fever, gastrointestinal manifestations (e.g., abdominal pain, vomiting, and nausea), central nervous syndromes (CNS) manifestations (e.g., dizziness, headache, encephalitis, and coma), diarrhoea, and haemorrhagic signs, among which CNS manifestations (adjusted odds ratio [OR] 30.26) are important risk factors for the death outcome of SFTS patients $[15,16]$. The blood- cerebrospinal fluid (CSF) barrier is an important barrier to protect the CNS from pathogens, and formed by a single layer of epithelial cells of the choroid plexus[17]. CSF provides nutrients, hormones, and signaling molecules to the brain, which is necessary for maintaining the normal function of the brain[18]. However, many clinical studies detected SFTSV RNA in patients' CSF samples and even successfully isolated SFTSV [19-21], which supported that the presence of virus in the CNS. All these findings point to SFTSV has the ability to damage the CNS, but there is limited research focus on the mechanism of SFTSV damage to the brain. This study detected the dynamic changes of viral load 
after SFTSV entering the brain and the target cells to deepen the understanding of the CNS manifestations caused by SFTSV.

\section{Materials And Methods}

\section{Ethics statement}

The study was reviewed and approved by the ethics committees of Shandong University. All procedures involving animals in this study were in accordance with the guidelines of the National Institutes of Health and ARRIVE guidelines (https://arriveguidelines.org).

Viruses and cells

The SFTSV strain JS2011-013-1(GenBank: KC505126 to KC505128) was used in this study. TCID ${ }_{50}$ was determined using methods described previously[14].

\section{Mouse infection experiments}

C57BL/ 6 pregnant mice were purchased from the experimental Animal Center of China Three Gorges University. Pregnant mice were raised under suitable conditions to normal delivery. One-day-old newborn mice were divided into 9 groups ( $n=6$ to 7 ). The newborn mice were injected intraperitoneally with the same amount of SFTSV $\left(6 \times 10^{3} \mathrm{TCID}_{50}\right)$ and six mock-infected mice were used in parallel as controls. At each time point of $1 \mathrm{~h}, 3 \mathrm{~h}, 6 \mathrm{~h}, 12 \mathrm{~h}, 24 \mathrm{~h}, 2$ days, 5 days, and 7 days after infection, the mice were sacrificed and their skulls were opened and brain tissues were taken out and frozen at $-80^{\circ} \mathrm{C}$. the viral load and infectious SFTSV in brain tissue were tested by qRT-PCR and IFA.

\section{Viral load determination by quantitative real-time PCR}

Frozen brain tissue was homogenized with zirconia beads in Tissuelyser II (Qiagen). The viral RNA in brain tissue was extracted using Total RNA Kit I (OMEGA, Guangzhou, China) and cDNAs were generated by RT First Strand cDNA Synthesis Kit (Servicebio, Wuhan, China). Viral load was determined by quantitative Real-time PCR (qRT-PCR) with an M segment-based SFTSV-specific primer set (SFTSV-MF: AAGAAGTGGCTGTTCATCATTATTG and SFTSV-MR: GCCTTAA GGACATTGGTGAGTA). The viral load was calculated as a multiple value and expressed as log2.

\section{Detection of infectious SFTSV in the brain tissue}

Frozen brain tissue was homogenized in $150 \mathrm{ml}$ DMEM, centrifuged at $15000 \mathrm{~g}$ for 10 minutes at $4^{\circ} \mathrm{C}$, and $100 \mathrm{ml}$ of supernatant was taken as SFTSV stock. The SFTSV stock was incubated with a monolayer of Vero cells for 2 hours in a 96-well plate, and then replaced with a medium with FBS (Gibco) concentration of $0.5 \%$ for 72 hours. Fix the cells with $4 \%$ paraformaldehyde, wash three times with PBS for 5 minutes each. Then, the monolayers were incubated for $1 \mathrm{~h}$ with human anti-SFTSV nucleoprotein (NP) mAb. After 
three 5-min washes with PBS, the monolayers were stained with FITC-conjugated goat anti- human IgG $(\mathrm{H}-\mathrm{L})$.

\section{Tissue immunofluorescence}

After 7 days of SFTSV infection, the brain tissues of newbron mice were submerged for 24 hours in $4 \%$ PFA at $4^{\circ} \mathrm{C}$. Tissues were paraffin embedded, and $4 \mu \mathrm{m}$-thick tissues sections were processed for tissue immunofluorescence. After blocking using 10\% BSA in assay buffer, primary and secondary antibodies were sequentially incubated with sections. SFTSV nucleoprotein (NP) human mAb and FITC-conjugated goat anti-human IgG (H-L) (proteintech, Chicago, USA) were used to probe SFTSV. Neun Rabbit mAb(CST, Boston, USA) and Cy3 conjugated Donkey Anti-Rabbit lgG $(\mathrm{H}+\mathrm{L})$ were used to probe neurons. F4/80 Rabbit mAb(CST, Boston, USA) and Cy3 conjugated Donkey Anti-Rabbit IgG $(\mathrm{H}+\mathrm{L})$ were used to probe macrophages. Nucleus were stained with DAPI (blue).

\section{Results}

In this study, the weight of newbron mice infected with SFTSV was not significantly abnormal (Fig. 1A). The qRT-PCR results showed that the viral RNA was detected in the brain tissue of newbron mice after intraperitoneal injection of SFTSV 3h (Fig. 1B). In addition, by detecting the viral load at different time points after SFTSV infection, we found that the viral load of SFTSV reached the peak at 6 h post-infection (hpi), decreased from 6 hpi to 24 hpi. Notably, viral load of SFTSV increased exponentially from $24 \mathrm{hpi}$ (Fig. 1B). The increase rate of viral load index in brain tissue of mice 24 hpi after SFTSV infection suggested that SFTSV may replicate and proliferate in brain tissue. Indirect immunofluorescence (IFA) showed that there were infectious SFTSV particles in the brain of SFTSV infected newbron mice (Fig. 1C), which further confirmed that SFTSV could break through the blood-brain barrier and enter the brain tissue.

In order to further determine the cell type of SFTSV infected brain tissue, we performed immunofluorescence analysis on mouse brain tissue at $7 \mathrm{dpi}$. The results showed that SFTSV infected neurons in cerebellum and midbrain, as well as macrophages in cerebellum, midbrain, hindbrain and thalamus(Fig. 2A and 2B). The local enlarged view further confirmed that neurons and macrophages were the target cells of SFTSV invading brain tissue. These findings indicated that SFTSV infected macrophages and neurons and replicated in the brain.

\section{Discussion}

Previous studies showed that no matter which routes adult mice were infected with SFTSV, they did not show clinical features, but some newborn mice will die after infection with high-dose SFTSV[22]. Therefore, in this study, we selected more susceptible newborn mice as the experimental animal.

The CNS manifestations caused by viral infection have always been a major public health issue of concern around the world because of the more serious clinical manifestations and even death[23]. It is 
known that a variety of viruses can invade the brain and cause encephalitis, including zika virus, Japanese encephalitis virus, tick-borne encephalitis virus, and SARS-CoV-2, etc[24-27]. A retrospective study of 214 COVID-19 patients in China showed that about $36.4 \%$ of patients showed nervous system symptoms, and a higher proportion of severe infection patients had CNS symptoms[28].

Since the discovery of SFTSV, people have noticed the CNS manifestations of SFTS patients, and found evidence of imaging and laboratory tests, which confirmed that SFTSV is capable of infecting the CNS $[19,29]$. However, due to the difficulty of collecting CNS-related clinical samples, the dynamic changes of viral load and target cell types after SFTSV infection in the brain are currently unclear.

We clarified the dynamic change of the viral load of SFTSV after invading the brain tissue. SFTSV broke through the blood-brain barrier (BBB) of newborn mice within $3 \mathrm{hpi}$. Considering the species differences between human and newborn mice, and adults have a fully functional BBB, it may take longer for SFTSV to break through the adult BBB[30]. After $24 \mathrm{hpi}$, the viral load increased exponentially in brain, which may be closely related to the differentiation of macrophages into the M2 phenotype. Previous studies found that SFTSV infected and replicated in macrophages in vivo and in vitro, and aid in virus shedding and spread by driving the differentiation of macrophages into the M2 phenotype[31, 32]. Our study reported that in addition to macrophages, SFTSV also infected neurons in the brain. Then, considering the complex and three-dimensional structure of the brain[33], there may be other cell types infected with SFTSV.

Our findings provide pathological evidence for the appearance of CNS manifestations in SFTS patients, and emphasize the urgency of exploring the molecular mechanisms underlying CNS manifestations.

\section{Declarations}

\section{Declaration of Competing Interest}

The authors have declared that no competing interests exist.

\section{Ethics approval and consent to participate}

All animal experimental procedures were approved by the ethics committees of Shandong University

\section{Consent for publication}

Not applicable.

\section{Availability of data and materials}

The datasets used and/or analysed during the current study are available from the corresponding author on reasonable request.

\section{Funding}


This work was funded by grants from Shandong Science and TechnologyDevelopment Plan. The funders had no direct influence over the present study.

\section{Authors' contributions}

Rui Chen and Hongling Wen: conceptualization and experiment design; Rui Chen, Qiang Li, Hongmei Chen, Hongguang Yang, and Xuemin Wei: experiment and data analysis; Mengting Chen and Xuemin Wei: investigation; Rui Chen: manuscript writing. All authors reviewed and agreed to the published version of the manuscript.

\section{References}

[1].Mansfield, K.L., et al., Emerging Tick-Borne Viruses in the Twenty-First Century. Front Cell Infect Microbiol, 2017. 7: p. 298.

[2].Paules, C.I., et al., Tickborne Diseases - Confronting a Growing Threat. N Engl J Med, 2018. 379(8): p. 701-703.

[3].Yu, X.J., et al., Fever with thrombocytopenia associated with a novel bunyavirus in China. $\mathrm{N}$ Engl $\mathrm{J}$ Med, 2011. 364(16): p. 1523-32.

[4].Tran, X.C., et al., Endemic Severe Fever with Thrombocytopenia Syndrome, Vietnam. Emerg Infect Dis, 2019. 25(5): p. 1029-1031.

[5].Kim, Y.R., et al., Severe Fever with Thrombocytopenia Syndrome Virus Infection, South Korea, 2010. Emerg Infect Dis, 2018. 24(11): p. 2103-2105.

[6].Takahashi, T., et al., The first identification and retrospective study of Severe Fever with Thrombocytopenia Syndrome in Japan. J Infect Dis, 2014. 209(6): p. 816-27.

[7].Li, H., et al., Clinical effect and antiviral mechanism of T-705 in treating severe fever with thrombocytopenia syndrome. Signal Transduct Target Ther, 2021. 6(1): p. 145.

[8].Luo, L.M., et al., Haemaphysalis longicornis Ticks as Reservoir and Vector of Severe Fever with Thrombocytopenia Syndrome Virus in China. Emerg Infect Dis, 2015. 21(10): p. 1770-6.

[9].Rainey, T., et al., Discovery of Haemaphysalis longicornis (Ixodida: Ixodidae) Parasitizing a Sheep in New Jersey, United States. Journal of Medical Entomology, 2018. 55(3): p. 757-759.

[10].Fang, X., et al., Epidemiological and clinical characteristics of severe fever with thrombocytopenia syndrome bunyavirus human-to-human transmission. PLOS Neglected Tropical Diseases, 2021. 15(4): p. e0009037. 
[11].Ye, C. and R. Qi, Risk factors for person-to-person transmission of severe fever with thrombocytopenia syndrome. Infect Control Hosp Epidemiol, 2021. 42(5): p. 582-585.

[12].Akagi, K., et al., Detection of viral RNA in diverse body fluids in an SFTS patient with encephalopathy, gastrointestinal bleeding and pneumonia: a case report and literature review. BMC Infectious Diseases, 2020. 20(1).

[13].Koga, S., et al., Severe Fever with Thrombocytopenia Syndrome Virus RNA in Semen, Japan. Emerg Infect Dis, 2019. 25(11): p. 2127-2128.

[14].Chen, R., et al., Severe fever with thrombocytopenia syndrome virus infection during pregnancy in C57/BL6 mice causes fetal damage. PLoS Negl Trop Dis, 2020. 14(7): p. e0008453.

[15].Li, H., et al., Epidemiological and clinical features of laboratory-diagnosed severe fever with thrombocytopenia syndrome in China, 2011-17: a prospective observational study. Lancet Infect Dis, 2018. 18(10): p. 1127-1137.

[16].Wang, X., et al., Clinical manifestations of death with severe fever and thrombocytopenia syndrome: A meta-analysis and systematic review. Journal of Medical Virology, 2020.

[17].Ghersi-Egea, J.F., et al., Molecular anatomy and functions of the choroidal blood-cerebrospinal fluid barrier in health and disease. Acta Neuropathol, 2018. 135(3): p. 337-361.

[18].Pellegrini, L., et al., Human CNS barrier-forming organoids with cerebrospinal fluid production. Science, 2020. 369(6500): p. eaaz5626.

[19].Wang, C., et al., Genome-based analysis of SFTSV causing severe encephalitis with brain lesions. J Neurovirol, 2020. 26(2): p. 181-187.

[20].Cui, N., et al., Severe fever with thrombocytopenia syndrome bunyavirus-related human encephalitis. J Infect, 2015. 70(1): p. 52-9.

[21].Kim, U.J., et al., Case report: detection of the identical virus in a patient presenting with severe fever with thrombocytopenia syndrome encephalopathy and the tick that bit her. BMC Infectious Diseases, 2018. 18(1).

[22].Chen, X.P., et al., Infection and pathogenesis of Huaiyangshan virus (a novel tick-borne bunyavirus) in laboratory rodents. J Gen Virol, 2012. 93(Pt 6): p. 1288-1293.

[23].Venkatesan, A. and O.C. Murphy, Viral Encephalitis. Neurol Clin, 2018. 36(4): p. 705-724.

[24].Rifino, N., et al., Neurologic manifestations in 1760 COVID-19 patients admitted to Papa Giovanni XXIII Hospital, Bergamo, Italy. Journal of Neurology, 2021. 268(7): p. 2331-2338. 
[25].Brugières, G.C.M.M., Zika Virus Associated with Meningoencephalitis. New England Journal of Medicine, 2016. 374(16): p. 1592-1595.

[26].Kuwata, R., et al., Distribution of Japanese Encephalitis Virus, Japan and Southeast Asia, 20162018. Emerging Infectious Diseases, 2020. 26(1): p. 125-128.

[27].Taba, P., et al., EAN consensus review on prevention, diagnosis and management of tick-borne encephalitis. European journal of neurology, 2017. 24(10): p. 1214-e61.

[28].Mao, L., et al., Neurologic Manifestations of Hospitalized Patients With Coronavirus Disease 2019 in Wuhan, China. JAMA Neurol, 2020. 77(6): p. 683-690.

[29].Kaneko, M., et al., A patient with severe fever with thrombocytopenia syndrome and hemophagocytic lymphohistiocytosis-associated involvement of the central nervous system. J Infect Chemother, 2018. 24(4): p. 292-297.

[30].Montagne, A., et al., Blood-brain barrier breakdown in the aging human hippocampus. Neuron, 2015. 85(2): p. 296-302.

[31].Suzuki, T., et al., Severe fever with thrombocytopenia syndrome virus targets B cells in lethal human infections. J Clin Invest, 2020. 130(2): p. 799-812.

[32].Zhang, L., et al., Severe Fever With Thrombocytopenia Syndrome Virus-Induced Macrophage Differentiation Is Regulated by miR-146. Front Immunol, 2019. 10: p. 1095.

[33].Hoffmann, J. and P.R. Holland, Brain structure and function related to headache. Cephalalgia, 2019. 39(13): p. 1603-1605.

\section{Figures}



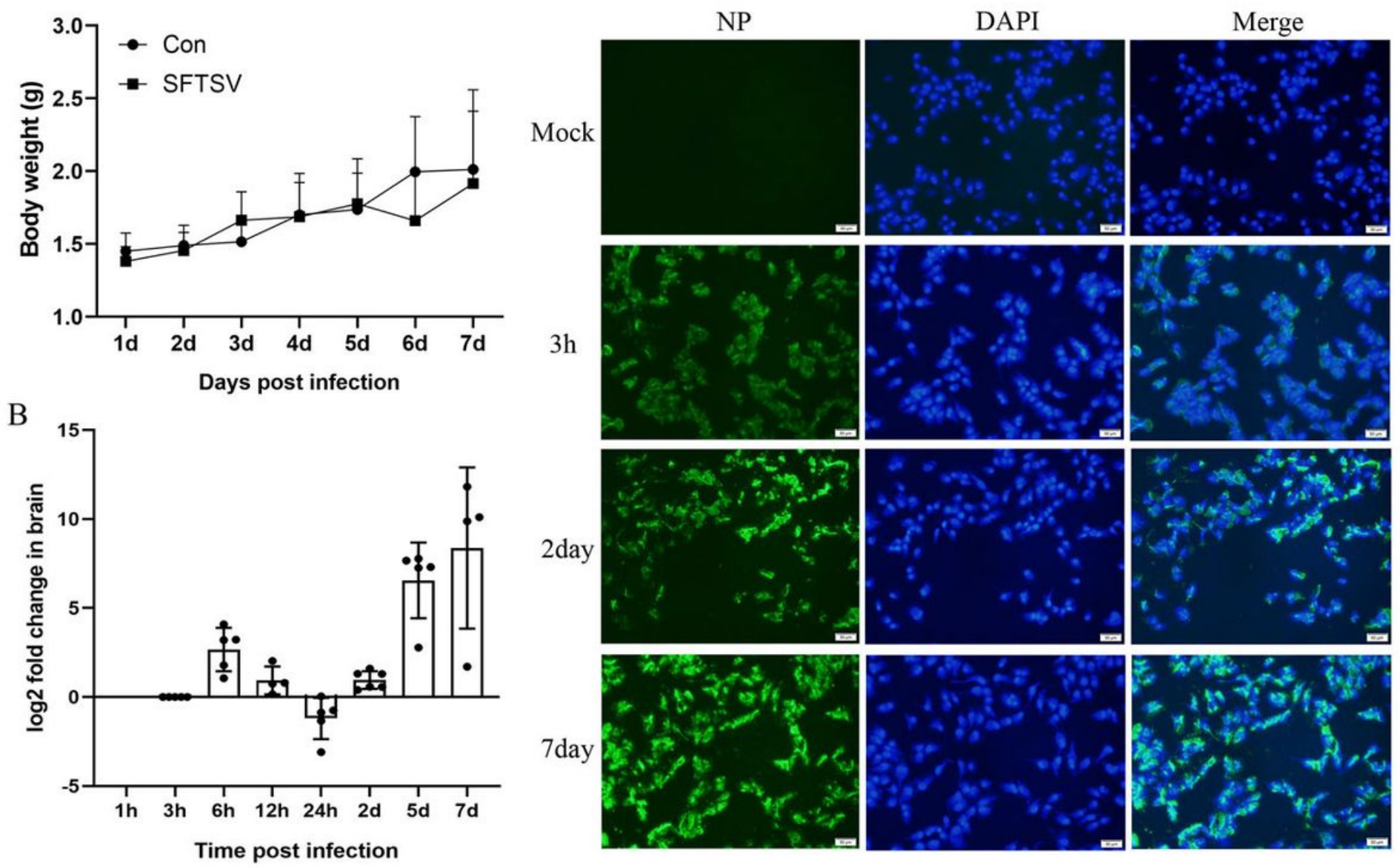

\section{Figure 1}

SFTSV replicates in brain tissue. A. Weight change of newborn mice after infection with SFTSV $(n=4$ to 6). B. Changes of viral load in brain tissues of newborn mice infected with SFTSV ( $n=4$ to 6$)$. qRT-PCR was used to detect the viral load in the brain tissue of newborn mice, and the SFTSV nucleic acid was detected at $3 \mathrm{hpi}$. The viral load of the $3 \mathrm{~h}$ group as a benchmark, the log 2 fold change of the other groups and the $3 \mathrm{~h}$ group was calculated. C. Indirect immunofluorescence (IFA) detected the infectious virus particles in the brain tissue. Newbron mice were sacrificed at 3 hpi, 2 day post-infection (dpi) and $7 \mathrm{dpi}$ respectively. The brain tissue was homogenized, and the infectious SFTSV particles were detected by IFA. SFTSV nucleoprotein (NP) human mAb labeled SFTSV followed by a FITC-conjugated goat anti-human IgG $(H+L)$ secondary antibody (green); nucleus were stained with DAPI (blue). Bar=50mm.

\section{Figure 2}

The target cells of SFTSV in the brain tissue of newborn mice. A. colocalization of SFTSV, and neurons in the brain tissue of newborn mice. a and $c$ indicated that SFTSV infected neurons in the cerebellum; $b$ and $d$ indicated that SFTSV infected neurons in the midbrain. NeuN, a marker protein of mature neurons, was labeled red. B. Colocalization of SFTSV, and macrophage in in brain tissue of newborn mice. a-d 
respectively indicated that SFTSV infected macrophages in the cerebellum, midbrain, hindbrain, and thalamus. The marker protein $\mathrm{F} 4 / 80$ of macrophages is marked in red. Nucleus were stained with DAPI (blue); SFTSV NP were marked Green. Bar=50mm. 